\title{
Über die Orte der Punkte, aus welchen ein Kreis durch spezielle Kegel projiziert wird.
}

\author{
Von L. Klug in Klausenburg.
}

1. Legt man durch einen Kreis $k$ den Rotationszylinder und die Kugel, von welcher $k$ ein Hauptkreis ist, so wird bekanntlich $k$ aus den Punkten des Zylinders mittels orthogonaler Kegel, und aus den Punkten der Kugel mittels Pappusscher Kegel projiziert. Wir suchen im folgenden den Ort der Punkte, aus welchen der Kreis durch andere spezielle Kegel projiziert wird.

2. Schreibt man dem Kreise $k$ das spitzwinklige Dreieck $A B C$ ein und legt durch die Seiten derselben die Flächen eines orthogonalen Dreikantes, so fällt die orthogonale Projektion der Spitze $M$ desselben in den Höhenpunkt $H$ des Dreieckes. Die Höhe $C H$ trifft den Kreis in dem zu $H$ in bezug auf die Seite $A B$ sym. metrischen Punkte $E$. Bezeichnet man mit $N$ einen Treffpunkt der Geraden $M H$ und derjenigen Kugel, von welcher $k$ ein Hauptkreis ist, und mit $D$ den Treffpunkt der Geraden $C E, A B$, so ist da $H E=2 H D$ wegen der rechtwinkligen Dreiecke $C N E, C M D$

$$
\bar{H}^{2}: \overline{H M}^{2}=C H . H E: C H . H D=2: 1 \text {. }
$$

Man erhält daher den Punkt $M$ auf der zur Kreisebene senkrechten halben Sehne $N H$ der Kugel, indem man diese im Verhältnis von $\sqrt{2}: 1$ verkürzt. Nun wird $k$ aus dem Punkte $M$ durch einen solchen Kegel $M . k$ projiziert, in welchen man ein, daher unendlich viele orthogonale Dreikante einschreiben kann; also ist $M . k$ ein gleichseitiger Kegel. Daraus folgt:

Der Ort der Punkte, aus welchen der Kreis $k$ vom Radius $r$ durch gleichseitige Kegel projiziert wird, ist ein Sphäroid (Rotationsellipsoid); der Äquator desselben ist $k$ und seine Rotationsachse ist gleich $r: \sqrt{2}$.

3. Es sei jetzt $A_{1} A_{2} A_{3}$ ein dem Kreise $k$ umschriebenes spitzwinkliges Dreieck und $M$ die Spitze eines der orthogonalen Dreikante, welche jenes Dreieck projizieren. Bezeichnet man mit $a_{i}$ den Winkel, welchen die Kante $M A_{i}$ mit der Kreisebene bildet, mit $K_{i}$ die orthogonale Projektion des Kreismittelpunk- 
tes $K$ auf jene Kante und mit $r$ den Radius des Kreises, so ist $r \cos a_{i}=M K_{i}$ und

$\overline{M K}^{2}=\overline{M K}_{1}^{2}+\overline{M K}_{2}^{2}+\overline{M K}_{3}^{2}=r^{2}\left(\cos ^{2} a_{1}+\cos ^{2} a_{2}+\cos ^{2} a_{3}\right)=2 r^{2}$,

d. h. $M$ hat den Abstand $r \sqrt{2}$ von $K$.

Nun wird $k$ aus dem Punkte $M$ durch einen Kegel $M . k$ projiziert, um welchen man ein und daher unendlich viele orthogonale Dreikante umschreiben kann; $M . k$ ist daher der reziproke des gleichseitigen Kegels. Wir haben also:

Der Ort der Punkte, aus welchen der Kreis $k$ vom Radius $r$ durch Kegel projiziert wird, welche dem gleichseitigen reziprok sind, ist eine mit $k$ konzentrische Kugel vom Radius $r \sqrt{\zeta}$.

Anmerkung. Betrachtet man $k$ als ein unendlich dünnes Ellipsoid, so ist jene Kugel die sogenannte Mongesche Kugel dieses Ellipsoids, welche die Eigensehaft hat, daf man aus ihren Punkten orthogonale Dreikante zum Ellipsoid legen kann.

4. Wir nehmen zwei Kreise $k$ und $l$ an, welche sich im Punkte $A$ berühren and deren Ebenen aufeinander senkrecht stehen und legen durch dieselben den Kegel, dessen Spitze $M$ sei. Bezeichnen $B$ und $C$ die zweiten Endpunkte der durch $A$ gehenden Diameter der Kreise. $k$ und $l$, so sind wegen der antiparallelen Kreisschnitte des Kegels die Winkel $M B A, M A C$ gleich. Verändert man daher der Größe nach den Diameter $A C$ von $l$, während $A$ fest bleibt, so beschreiben die Strahlen $B M, A M$ zwei gleiche sich entgegengesetzt drehende Strahlenbüschel um $B$ und $A$, und $M$ beschreibt in der Ebene $A B C$ eine gleichseitige Hyperbel, von welcher $A B$ die reelle Achse ist. Aus den Punkten dieser Hyperbel wird also der feste Kreis $k$ durch solche Kegel projiziert, deren zyklische Ebenen orthogonal sind and die man daher orthozyklisch nennen kann. Also:

Der Ort der Punkte, aus welchen der $K r$ is $k$ durch orthozyklische Kegel projiziert wird, ist ein Rotationshyperboloid; die Meridiane derselben sind gleichseitige Hyperbeln und sein Kehlkreis ist $k$ selbst.

5 . Der reziproke Kegel des Pap pusschen ist der Hachet tesche. Derselbe hat die Eigenschaft, daß die Polarebene eines Fokalstrahles senkrecht steht auf dem anderen Fokalstrahl. Gestützt auf diese Eigenschaft, wollen wir durch den Kreis $k$ vom Radius $r$ einen $\mathrm{H}$ a chett eschen Kegel legen.

Es seien $K F H G$ die Eckpunkte, $O$ der Mittelpunkt eines innerhalb $k$ liegenden Quadrates, dessen Eckpunkt $K$ im Mittelpunkte von $k$ ist. Die Polare von $F$ treffe die Seiten $K F, G H$ und die Diagonale $K H$ des Quadrates in den Punkten $E, I$ und $J$; die Seite $F H$ treffe den Kreis im Punkte $L$, und die mit $F^{\prime} L$ 
gleiche Strecke $H M$ stehe im Punkte $H$ senkrecht zur Kreisebene. Da

$$
\widetilde{H M}^{2}=\overline{F L}^{2}=K F \cdot F E=O H . H J
$$

ist, so bilden die Geraden $M O, M J$ and die Ebenen $M F O, M F J$ rechte Winkel; da ferner die konjugierten Polarpaare $F K, F H$; $F O, F J$ aus der Geraden $M F$ durch rechtwinklige Ebenenpaare projiziert werden, so ist die Gerade $M F$ ein Fokalstrahl desjenigen Kegels $M . k$, welcher den Kreis $k$ aus dem Punkte $M$ projiziert. $\mathrm{Da}$ nun der andere Fokalstrahl $M G$ dieses Kegel wegen $H M^{2}=$ $K F \cdot F E=G H . H I$ auf die Polarebene $M E F$ des ersteren Fokalstrahles senkrecht steht, so ist $M . K$ ein $\mathrm{Hachette} \mathrm{scher} \mathrm{Kegel.}$

Dreht man den Kreis $k$ um $90^{\circ}$ um die $K F$ und projiziert ihn dann mit zu $F H$ parallelen Strahlen auf die Ebene $\dot{K} H M$, so bildet diese Projektion den Ort der Punkte $\boldsymbol{M}$ in dieser Ebene. Daraus folgt:

Der Ort der Punkte, aus welchen ein Kreis $k$ durch Hachettesche Kegel projiziert wird, ist ein Sphäriod; seine Rotationsachse ist gleich dem Diameter des Kreises $k$ und sein Fokalkreis ist $k$.

6. Suchen wir jetzt den Ort der Punkte, aus welchen $k$ durch Kegel mit orthogonalen Fokalstrahlen (orthofokale Kegel) projiziert wird.

Es sei $J$ ein in der Kreisebene $*$ liegender Punkt, welcher vom Kreismittelpunkte $K$ einen solchen Abstand hat, wie die Seite eines dem Kreise einbeschriebenen Quadrates. Dann halbiert die Polare $F^{\prime} F^{\prime}$ von $J$ in bezug auf $k$ im Schnittpunkte $G$ die Strecke $K J$. Wir beschreiben über $G J$ als Durchmesser einen Kreis $l$, dessen Ebene auf $x$ senkrecht steht, nehmen auf $l$ einen Punkt $M$ and auf die Polare die Punkte $F F^{\prime \prime}$ so an, dals $G M=G F^{\prime}=F^{\prime} G$ sei und bezeichnen die orthogonale Projektion von $M$ auf $G J$ mit $H$. $\mathrm{Da}$

$$
\overline{G F}^{2}=\overline{G M}^{2}=G H \cdot G J=G H . K G
$$

ist, so ist der Winkel $K F H$ ein rechter, und es werden daher aus $M$ die konjugierten Polarpaare $F K, F H ; F G, F J$ durch orthogonale Ebenenpaare und also $k$ durch einen Kegel $M . k$ projiziert, von welchem $M F, M F^{\prime \prime}$ Fokalstrahlen sind. Diese Fokalstrahlen stehen aber senkrecht aufeinander, daher ist $M . k$ ein orthofokaler Kegel.

Da man nun den Punkt $M$ auf dem Kreis $i$ beliebig annehmen und diesen Kreis um die zur Ebene $x$ im Mittelpunk te $K$ senkrecht stehende Achse drehen kann, so folgt:

Der Ort der Punkte, aus welchen ein Kreis $k$ vom Radius r durch orthofokale Kegel projiziert wird, ist eine Ringfläche; der Äquator und der Kehlkreis desselben ist mit $k$ konzentrisch und liegt in 
der Kreisebene und die Radien dieser Kreisesind $r \sqrt{2}$ und $r: \overline{2}$.

Anmerkung. Bilden die Fokalstrahlen statt einen Winkel von $2 \varphi$, so iubergeht jene Ringfläche in eine andere. Der Radius des Kehlkreises und des Äquators derselben ist $r \sin \varphi$, bezw. $r: \sin \varphi$.

7. Der reziproke Kegel des orthogonalen hat die Eigenschaft, daß ihn die zu den Fokalstrahlen senkrechten Ebenen in Parabeln schneiden. $\mathrm{Da}$ sich daraus viele Eigensehaften der $\mathrm{Pa}$ rabel auf den Kegel übertragen lassen, so wollen wir ihn parabolischen Kegel nennen.

Um nun den Ort zu finden, aus dessen Punkten der Kreis $k$ durch parabolische Kegel projiziert wird, sei $E F$ die Polare eines außerhalb $k$ liegenden Punktes $J$, weleher den Kreis $k$ vom Mittelpunkte $K$ in einem $E$ Punkte treffe. Ferner sei $K F \perp E K$ und $G$ der Treffpunkt von $E F, K J$; der Punkt $H$ sei auf $\overline{K J}$ so gelegen, daß $F H \perp K F$; der Punkt $M$ auf der zur Kreisebene im Punkte $H$ senkrecht stehenden Geraden so angenommen, daß $K G=G M$; schließlich sei $\Varangle M K J=\varphi$, also $M G J=2 \varphi$.

Da infolge der ähnlichen Dreiecke $H F K, K E J$

$$
H G: G K=G K: G J
$$

also $H G . G J=\overline{G M}^{2}$ ist, so stehen die Geraden $G M, M J$, wie auch die Ebenen $M G F, M J F$ senkrecht aufeinander, und es werden daher die konjugierten Polarpaare $K F, F H ; G F, F J$ aus dem Punkte $M$ durch orthogonale Ebenenpaare projiziert. Da ferner die Geraden $F M$ zur Kreistangente $E J$ und zur Geraden $M E$ senkrecht steht, so steht sie auch senkrecht zur Ebene MEJ. Daraus folgt, daß der den Kreis aus dem Punkte $M$ projizierende Kegel $M . K$ ein parabolischer ist, da sein Fokalstrahl $F M$ zur 'Tangentialebene $M E J$ des Kegels senkrecht steht.

Um nun den Ort der Punkte $M$ in der Ebene $K M J$ zu finden, wenn sich $J$ auf dem Kreisdurchmesser bewegt, beachte man, daß infolge der kongruenten Dreiecke $E K F, E M F$

ferner

$$
\bar{K} \bar{M}^{2}=2 . \bar{K} \bar{F}^{2}
$$

$$
\frac{\overline{K F}^{2}}{\overline{K E}^{2}}=\frac{\overline{H F}^{2}}{\overline{K F}^{2}}=\frac{H G}{G K}=\frac{H G}{M G}=\cos 2 \varphi
$$

woraus

$$
\overline{K M}^{2}=2 \cdot \overline{K E}^{2} \cdot \cos 2 \varphi .
$$

Das ist aber die Polargleichung einer Lemniscate, deren Brennpunkte die Endpunkte des Kreisdurchmessers $K J$ sind. Wir haben daher:

Der Ort der Punkte, aus welchen der Kreis durch parabolische Kegel (dem orthogonalen reziproken Kegel) projiziert wird, ist eine Rotationsfläche; die Meridiane derselben sind schlichte Lemniscaten, deren Brennpunkte in den Endpunkten der Kreisdurchmesser liegen. 